PROCEEDINGS OF THE

AMERICAN MATHEMATICAL SOCIETY

Volume 134, Number 7 , Pages 2153-2159

S 0002-9939(05)08177-3

Article electronically published on December 19, 2005

\title{
THE BERRY-ESSEEN BOUND FOR CHARACTER RATIOS
}

\author{
QI-MAN SHAO AND ZHONG-GEN SU \\ (Communicated by Richard C. Bradley)
}

\begin{abstract}
Let $\lambda$ be a partition of $n$ chosen from the Plancherel measure of the symmetric group $S_{n}$, let $\chi^{\lambda}(12)$ be the irreducible character of the symmetric group parameterized by $\lambda$ evaluated on the transposition (12), and let $\operatorname{dim}(\lambda)$ be the dimension of the irreducible representation parameterized by $\lambda$. Fulman recently obtained the convergence rate of $O\left(n^{-s}\right)$ for any $0<s<\frac{1}{2}$ in the central limit theorem for character ratios $\frac{(n-1)}{\sqrt{2}} \frac{\chi^{\lambda}(12)}{\operatorname{dim}(\lambda)}$ by developing a connection between martingale and character ratios, and he conjectures that the correct speed is $O\left(n^{-1 / 2}\right)$. In this paper we confirm the conjecture via a refinement of Stein's method for exchangeable pairs.
\end{abstract}

\section{INTRODUCTION AND MAIN RESULT}

Let $n \geq 1$, let $\lambda=\left(\lambda_{1}, \lambda_{2}, \cdots, \lambda_{p}\right)$ be a partition of $n$, i.e., $\lambda_{1}+\lambda_{2}+\cdots+\lambda_{p}=n$, and write simply $\lambda \vdash n$. Denote by $\operatorname{dim}(\lambda)$ the number of standard Young tableaux associated with the shape $\lambda$. By the Robinson-Schensted-Knuth correspondence [18, we have

$$
\sum_{\lambda \vdash n} \operatorname{dim}(\lambda)^{2}=n ! .
$$

Thus we produce the so-called Plancherel measure

$$
P(\{\lambda\})=\frac{\operatorname{dim}(\lambda)^{2}}{n !} .
$$

Recently there has been intensive interest in the statistical properties of partitions chosen from the Plancherel measure. We refer the reader to the surveys by Aldous and Diaconis [1], Defit [4] and the seminal papers of Borodin, Okounkov and Olshanski [2], Johansson [14], and Okounkov and Pandharipande [16] for details.

It turns out that the Plancherel measure can also be regarded as a probability measure on the irreducible representation of the symmetric group $S_{n}$. Observe that the irreducible representation of the symmetric group $S_{n}$ is parameterized by partitions $\lambda$ of $n$ and $\operatorname{dim}(\lambda)$ is just the corresponding dimension of the irreducible representation.

Received by the editors September 28, 2004 and, in revised form, February 4, 2005.

2000 Mathematics Subject Classification. Primary 60F05; Secondary 05E10, 60C05.

Key words and phrases. Berry-Esseen bound, character ratio, Plancherel measure, Stein's method.

The first author was supported in part by Grant R-1555-000-035-112 at the National University of Singapore.

The second author was supported in part by NFS of China (No. 10371109).

(C)2005 American Mathematical Society 
Let $\chi^{\lambda}(12)$ be the irreducible character parameterized by $\lambda$ evaluated on the transposition (12). The quantity $\frac{\chi^{\lambda}(12)}{\operatorname{dim}(\lambda)}$ is called a character ratio and is crucial for analyzing the convergence rate of the random walk on the symmetric group generated by transpositions in Diaconis and Shahshahani [5]. In fact, Diaconis and Shahshahani prove that the eigenvalues for this random walk are the character ratios $\frac{\chi^{\lambda}(12)}{\operatorname{dim}(\lambda)}$, each occurring with multiplicity $\operatorname{dim}(\lambda)^{2}$. Character ratios also play an essential role in work on the moduli spaces of curves; see Eskin and Okounkov [6], Okounkov and Pandharipande [16].

Kerov 15. first studies the asymptotic behavior for character ratios and outlines the proof of the following central limit theorem:

$$
\frac{(n-1)}{\sqrt{2}} \frac{\chi^{\lambda}(12)}{\operatorname{dim}(\lambda)} \stackrel{d}{\longrightarrow} N(0,1) .
$$

A full proof of the result appears in Ivanov and Olshanski [13] see also Hora 12 , for another proof. A more probabilistic approach to Kerov's central limit theorem has recently been given by Fulman [7, in which a Stein's method for exchangeable pairs is used to obtain for all $n \geq 2, z \in R$,

$$
\left|P\left(\frac{(n-1)}{\sqrt{2}} \frac{\chi^{\lambda}(12)}{\operatorname{dim}(\lambda)} \leq z\right)-\Phi(z)\right| \leq 40.1 n^{-1 / 4}
$$

where $\Phi(z)$ is the standard normal distribution function.

More recently Fulman [8] developed a connection between martingales and character ratios of the symmetric group, and thereby improved the above speed of convergence to $O\left(n^{-s}\right)$ for any $s<\frac{1}{2}$. He also conjectured that the correct speed is $O\left(n^{-1 / 2}\right)$.

The main aim of this note is to confirm the following conjecture.

Theorem 1.1. We have

$$
\sup _{z}\left|P\left(\frac{(n-1)}{\sqrt{2}} \frac{\chi^{\lambda}(12)}{\operatorname{dim}(\lambda)} \leq z\right)-\Phi(z)\right| \leq A n^{-1 / 2}
$$

where $A$ is an absolute constant.

The proof of Theorem 1.1 will be given in Section 2. The main technique is a refinement of Stein's method for exchangeable pairs (see Theorem 2.1 below). Recall that two random variables $W, W^{*}$ are called exchangeable if $\left(W, W^{*}\right)$ and $\left(W^{*}, W\right)$ have the same joint distribution function. In order to apply Stein's approach for exchangeable pairs, one needs to construct a $W^{*}$ such that $\left(W, W^{*}\right)$ is exchangeable and the difference $W-W^{*}$ is small. Fulman [7] uses the theory of harmonic functions on Bratelli diagrams and shows how it can be applied to generate a natural exchangeable pair $\left(W, W^{*}\right)$. The basic idea is to use a reversible Markov chain on the set of partitions of size $n$ whose stationary distribution is the Plancherel measure. Let $\lambda^{*}$ be obtained from $\lambda$ by one step in the chain, and then set $\left(W, W^{*}\right)=\left(W(\lambda), W^{*}(\lambda)\right)$. This construction also has the merit of being applicable to more general groups [9] and to measures arising from symmetric functions [10.

In the setting of Theorem 1.1, we let $W=\frac{(n-1)}{\sqrt{2}} \frac{\chi^{\lambda}(12)}{\operatorname{dim}(\lambda)}$. Let parents $(\lambda, \mu)$ denote the set of partitions above both $\lambda, \mu$ in the Young lattice (this set has size 0 or 1 
unless $\lambda=\mu$ ), i.e.,

$$
\operatorname{parents}(\lambda, \mu)=\#\{\tau: \lambda \nearrow \tau, \mu \nearrow \tau\}
$$

Define

$$
W^{*}(\lambda)=W\left(\lambda^{*}\right)
$$

where, given $\lambda$, the partition $\lambda^{*}$ is $\mu$ with probability

$$
J(\lambda, \mu)=\frac{\operatorname{dim}(\mu)|\operatorname{parents}(\lambda, \mu)|}{(n+1) \operatorname{dim}(\lambda)} .
$$

Then it follows from Proposition 2.1 of Fulman [7] that $\left(W, W^{*}\right)$ is an exchangeable pair.

\section{Proof}

The proof is based on the following refinement of Stein's result 20] for exchangeable pairs.

Theorem 2.1. Let $\left(W, W^{*}\right)$ be an exchangeable pair of real-valued random variables such that

$$
E^{W}\left(W^{*}\right)=(1-\tau) W
$$

with $0<\tau<1$, where $E^{W}\left(W^{*}\right)$ denotes the conditional expected value of $W^{*}$ given $W$. Assume $E\left(W^{2}\right) \leq 1$. Then for any $a>0$,

$$
\begin{aligned}
& \sup _{z}|P(W \leq z)-\Phi(z)| \\
& \leq \sqrt{E\left(1-\frac{1}{2 \tau} E^{W}\left(\Delta^{2}\right)\right)^{2}}+\frac{0.41 a^{3}}{\tau}+1.5 a+\frac{1}{2 \tau} E \Delta^{2} I_{\{|\Delta| \geq a\}},
\end{aligned}
$$

where $\Delta=W-W^{*}$.

If $\Delta$ is bounded, say $|\Delta| \leq a_{0}$ for a constant $a_{0}$, then (2.3) reduces to

$$
\sup _{z}|P(W \leq z)-\Phi(z)| \leq \sqrt{E\left(1-\frac{1}{2 \tau} E^{W}\left(\Delta^{2}\right)\right)^{2}}+\frac{0.41 a_{0}^{3}}{\tau}+1.5 a_{0} .
$$

Similar results for the bounded case were obtained by Rinott and Rotar [17] and Rinott and Goldstein [11.

Theorem 1.1 is an easy consequence of Theorem 2.1

Proof of Theorem 1.1. By [7, we can choose

$$
\tau=\frac{2}{n+1}, \quad \sqrt{E\left(1-\frac{1}{2 \tau} E^{W}\left(\Delta^{2}\right)\right)^{2}} \leq \frac{\sqrt{3}}{2 n^{1 / 2}} .
$$

Let $a=4 e \sqrt{2} n^{-1 / 2}$. Then, by the proof of Proposition 4.6 in [7],

$$
\begin{aligned}
E \Delta^{2} I_{\{|\Delta|>a\}} & \leq 8 P(|\Delta|>a) \\
& \leq 8 P\left(\max \left(\lambda_{1}, \lambda_{1}^{\prime}\right)>2 e \sqrt{n}\right) \\
& \leq 16 e^{-2 e \sqrt{n}}
\end{aligned}
$$

and hence

$$
\begin{aligned}
\frac{1}{2 \tau} E \Delta^{2} I_{\{|\Delta|>a\}} & \leq 4(n+1) e^{-2 e \sqrt{n}} \\
& \leq n^{-1 / 2} 4(n+1)^{3 / 2} e^{-2 e \sqrt{n}} \\
& \leq 0.05 n^{-1 / 2} .
\end{aligned}
$$


Therefore, by Theorem 2.1

$$
\begin{aligned}
\sup _{z} & |P(W \leq z)-\Phi(z)| \\
\leq & \frac{\sqrt{3}}{2 n^{1 / 2}}+0.205(n+1)(4 e \sqrt{2})^{3} n^{-3 / 2}+4 e \sqrt{2} n^{-1 / 2}+0.05 n^{-1 / 2} \\
\leq & A n^{-1 / 2}
\end{aligned}
$$

where $A$ is an absolute constant.

We remark that if one uses

$$
P\left(\lambda_{1} \geq k\right) \leq\left(\begin{array}{l}
n \\
k
\end{array}\right) / k !
$$

for $1 \leq k \leq n$ (see Lemma 1.4.1 in [19]) and chooses $a=\delta n^{-1 / 2}$ with $\delta>0$ properly, then the constant $A$ can be reduced to 150 .

Now we turn to the proof of Theorem 2.1.

Proof of Theorem 2.1. For any measurable function $f$ with $E\{(|W|+1)|f(W)|\}<$ $\infty$, exchangeability and (2.2) imply

$$
\begin{aligned}
0 & =E\left\{\left(W-W^{*}\right)\left(f(W)+f\left(W^{*}\right)\right)\right\} \\
& =2 E\left\{f(W)\left(W-W^{*}\right)\right\}+E\left\{\left(W-W^{*}\right)\left(f\left(W^{*}\right)-f(W)\right)\right\} \\
& =2 \tau E\{W f(W)\}-E\left\{\left(W-W^{*}\right)\left(f(W)-f\left(W^{*}\right)\right)\right\},
\end{aligned}
$$

and hence

$$
E\{W f(W)\}=\frac{1}{2 \tau} E\left\{\left(W-W^{*}\right)\left(f(W)-f\left(W^{*}\right)\right)\right\} .
$$

Now let $f=f_{z}$ be the solution of the following Stein equation:

$$
f_{z}^{\prime}(x)-x f_{z}(x)=I_{\{x \leq z\}}-\Phi(z) .
$$

It is known (see [20, p.22]) that $f$ is given by

$$
f_{z}(x)= \begin{cases}\sqrt{2 \pi} e^{x^{2} / 2} \Phi(x)[1-\Phi(z)] & \text { if } x \leq z, \\ \sqrt{2 \pi} e^{x^{2} / 2} \Phi(z)[1-\Phi(x)] & \text { if } x \geq z,\end{cases}
$$

satisfying

$$
\begin{gathered}
\left|x f_{z}(x)\right| \leq 1,0<f_{z}(x) \leq \sqrt{2 \pi} / 4, \\
\left|f_{z}^{\prime}(x)\right| \leq 1,\left|f_{z}^{\prime}(x)-f_{z}^{\prime}(y)\right| \leq 1 \\
\left|(x+u) f_{z}(x+u)-x f_{z}(x)\right| \leq(|x|+\sqrt{2 \pi} / 4)|u|
\end{gathered}
$$

for all real $x, y$, and $u$. For the proofs of the above inequalities, we refer to [20, p.23] for (2.6) and the first inequality of (2.7), and to Chen and Shao [3] for the second inequality of (2.7). (2.8) is a consequence of (2.6), (2.7) and the mean value theorem. 
By (2.5), we have

$$
\begin{aligned}
P(W \leq z)-\Phi(z)= & E f_{z}^{\prime}(W)-E W f_{z}(W) \\
= & E f_{z}^{\prime}(W)-\frac{1}{2 \tau} E\left\{\left(W-W^{*}\right)\left(f_{z}(W)-f_{z}\left(W^{*}\right)\right)\right\} \\
= & E\left\{f_{z}^{\prime}(W)\left(1-\frac{1}{2 \tau} \Delta^{2}\right)\right\} \\
& -\frac{1}{2 \tau} E\left\{\Delta\left(f_{z}(W)-f_{z}(W-\Delta)-\Delta f_{z}^{\prime}(W)\right)\right\} \\
:= & J_{1}+J_{2} .
\end{aligned}
$$

It follows from (2.6) that

$$
\begin{aligned}
\left|J_{1}\right| & =\left|E\left\{f_{z}^{\prime}(W)\left(1-\frac{1}{2 \tau} E^{W}\left(\Delta^{2}\right)\right)\right\}\right| \\
& \leq E\left|1-\frac{1}{2 \tau} E^{W}\left(\Delta^{2}\right)\right| \\
& \leq \sqrt{E\left(1-\frac{1}{2 \tau} E^{W}\left(\Delta^{2}\right)\right)^{2}} .
\end{aligned}
$$

To bound $J_{2}$, write

$$
\begin{aligned}
E\left\{\Delta\left(f_{z}(W)-f_{z}(W-\Delta)-\Delta f_{z}^{\prime}(W)\right)\right\} & \\
= & E\left\{\Delta \int_{-\Delta}^{0}\left(f_{z}^{\prime}(W+t)-f_{z}^{\prime}(W)\right) d t\right\} \\
= & E\left\{\Delta I_{\{|\Delta|>a\}} \int_{-\Delta}^{0}\left(f_{z}^{\prime}(W+t)-f_{z}^{\prime}(W)\right) d t\right\} \\
& +E\left\{\Delta I_{\{|\Delta| \leq a\}} \int_{-\Delta}^{0}\left(f_{z}^{\prime}(W+t)-f_{z}^{\prime}(W)\right) d t\right\} \\
& :=J_{2,1}+J_{2,2} .
\end{aligned}
$$

By (2.7),

$$
\left|J_{2,1}\right| \leq E \Delta^{2} I_{\{|\Delta|>a\}}
$$

Using (2.5) again, we have

$$
\begin{aligned}
J_{2,2}= & E\left\{\Delta I_{\{|\Delta| \leq a\}} \int_{-\Delta}^{0}\left((W+t) f_{z}(W+t)-W f_{z}(W)\right) d t\right\} \\
& +E\left\{\Delta I_{\{|\Delta| \leq a\}} \int_{-\Delta}^{0}\left(I_{\{W+t \leq z\}}-I_{\{W \leq z\}}\right) d t\right\} \\
:= & J_{2,2,1}+J_{2,2,2} .
\end{aligned}
$$

By (2.8),

$$
\begin{aligned}
\left|J_{2,2,1}\right| & \leq E\left\{\Delta I_{\{|\Delta| \leq a\}} \int_{-\Delta}^{0}(|W|+\sqrt{2 \pi} / 4)|t| d t\right\} \\
& \leq E\left\{0.5|\Delta|^{3} I_{\{|\Delta| \leq a\}}(|W|+\sqrt{2 \pi} / 4)\right\} \\
& \leq 0.5 a^{3}(\sqrt{2 \pi} / 4+E|W|) \\
& \leq 0.5 a^{3}(\sqrt{2 \pi} / 4+1) \leq 0.82 a^{3} .
\end{aligned}
$$


As for $J_{2,2,2}$, observe that

$$
\begin{aligned}
J_{2,2,2} & \leq E\left\{\Delta I_{\{0 \leq \Delta \leq a\}} \int_{-\Delta}^{0} I_{\{z \leq W \leq z-t\}} d t\right\} \\
& \leq E\left(\Delta^{2} I_{\{0 \leq \Delta \leq a\}} I_{\{z \leq W \leq z+a\}}\right) \\
& \leq 3 a \tau,
\end{aligned}
$$

where in the last inequality we used the concentration inequality in Lemma 2.1 below.

Similarly, we have

This proves Theorem 2.1.

$$
J_{2,2,2} \geq-3 a \tau .
$$

Lemma 2.1. Under the assumption of Theorem 2.1, we have

$$
E\left(\Delta^{2} I_{\{0 \leq \Delta \leq a\}} I_{\{z \leq W \leq z+a\}}\right) \leq 3 a \tau
$$

for $a>0$.

Proof. Let

$$
f(x)= \begin{cases}-1.5 a & \text { for } x \leq z-a \\ x-z-a / 2 & \text { for } z-a \leq x \leq z+2 a \\ 1.5 a & \text { for } x \geq z+2 a\end{cases}
$$

By (2.4),

$$
\begin{aligned}
3 a \tau & \geq 2 \tau E(W f(W)) \\
& =E\left\{\left(W-W^{*}\right)\left(f(W)-f\left(W^{*}\right)\right)\right\} \\
& =E\left\{\Delta \int_{-\Delta}^{0} f^{\prime}(W+t) d t\right\} \\
& \geq E\left\{\Delta \int_{-\Delta}^{0} I_{\{|t| \leq a\}} I_{\{z \leq W \leq z+a\}} f^{\prime}(W+t) d t\right\} \\
& =E\left(|\Delta| \min (a,|\Delta|) I_{\{z \leq W \leq z+a\}}\right) \\
& \geq E\left(\Delta^{2} I_{\{0 \leq \Delta \leq a\}} I_{\{z \leq W \leq z+a\}}\right)
\end{aligned}
$$

as desired.

\section{REFERENCES}

[1] Aldous, D. and Diaconis, P. (1999) Longest increasing subsequences: from patient sorting to Baik-Defit-Johansson theorem. Bull. AMS (N.S.) 36, 413-432. MR1694204 (2000g:60013)

[2] Borodin, A., Okounkov, A. and Olshanski, G. (2000). Asymptotics of Plancherel measures for symmetric groups. J. Amer. Math. Soc. 13, 481-515. MR.1758751 (2001g:05103)

[3] Chen, L.H.Y. and Shao, Q.M. (2001). A non-uniform Berry-Esseen bound via Stein's method. Probab. Theory Related Fields 120, 236-254. MR 1841329 (2002h:60037)

[4] Defit, P. (2000). Integrable systems and combinatorial theory. Notices Amer. Math. Soc. 47, 631-640. MR1764262 (2001g:05012)

[5] Diaconis, P. and Shahshahani, M. (1981). Generating a random permutation with random transpositions. Z.Wahr. Verw. Gebiete 57, 159-179. MR0626813 (82h:60024)

[6] Eskin, A. and Okounkov, A. (2001). Asymptotics of branched coverings of a torus and volumes of moduli spaces of holomorphic differentials. Invent. Math. 145, 59-103. MR 1839286 (2002g:32018)

[7] Fulman, J. (2005). Stein's method and Plancherel measure of the symmetric group. Trans. AMS 357, 555-570. 
[8] Fulman, J. (2004). Martingales and character ratios, preprint.

[9] Fulman, J. (2004). Card shuffling and the decomposition of tensor products.Pacific J. Math. 217 (2004), no. 2, 247-262. MR2109933

[10] Fulman, J. (2004). Stein's method, Jack measure and the metropolis algorithm, J. Combin. Theory, A. 108 (2004), no. 2, 275-296. MR2098845

[11] Goldstein, L. and Rinott, Y. (1996). Multivariate normal approximations by Stein's method and size bias couplings. J. Appl. Probab. 33, 1-17. MR.1371949 (97d:60038)

[12] Hora, A.(1998). Central limit thorem for the adjacency operators on the infinite symmetric group. Comm. Math. Phys. 195, 405-416. MR.1637801 (99i:46058)

[13] Ivanov, V. and Olshanski, G. (2002). Kerov's central limit theorem for the Plancherel measure on Young diagrams. Symmetric Functions 2001: Surveys of developments and perspectives, Kluwer Academic Publishers, Dordrecht. MR2059361 (2005d:05148)

[14] Johansson, K. (2001). Discrete orthogonal polynomial ensembles and the Plancherel measure. Ann. Math. 153, 259-296. MR1826414(2002g:05188)

[15] Kerov, S. V. (1993). Gaussian limit for the Plancherel measure of the symmetric group. Compt. Rend. Acad. Sci. Paris I, 303-308. MR1204294 (93k:20106)

[16] Okounkov, A. and Pandharipande, R. Gromov-Witten theory, Hurwitz numbers, and matrix models I, preprint.

[17] Rinott, Y. and Rotar, V. (1997). On coupling constructions and rates in the CLT for dependent summands with applications to the antivoter model and weighted $U$-statistics. Ann. Appl. Probab. 7, 1080-1105. MR1484798 (99g:60050)

[18] Sagan, B. (1991). The symmetric group. Representations, combinatorial algorithms, and symmetric functions. Springer-Verlag.

[19] Steele, J.M. (1997). Probability Theory and Combinatorial Optimization. Society for Industrial and Applied Mathematics, Philadelphia. MR.1422018 (99d:60002)

[20] Stein, C. (1986). Approximate computation of expectations. Institute of Mathematical Statistics, Lect. Notes 7. MR0882007 (88j:60055)

Department of Mathematics, University of Oregon, Eugene, Oregon 97403 - and Department of Mathematics, Department of Statistics and Applied Probability, NaTIONAL UNIVERSITY OF Singapore

E-mail address: qmshao@darkwing.uoregon.edu

Department of Mathematics, Zhejiang University, Hangzhou, Zhejiang 310027, PeoPLE's Republic of China 